\title{
Ranking-based Method for News Stance Detection
}

\author{
Qiang Zhang \\ University College London \\ London, United Kingdom \\ qiang.zhang.16@ucl.ac.uk
}

\author{
Emine Yilmaz \\ University College London \\ London, United Kingdom \\ emine.yilmaz@ucl.ac.uk
}

\author{
Shangsong Liang \\ KAUST \\ Thuwal, Saudi Arabia \\ shangsong.liang@kaust.edu.sa
}

\begin{abstract}
A valuable step towards news veracity assessment is to understand stance from different information sources, and the process is known as the stance detection. Specifically, the stance detection is to detect four kinds of stances ("agree", "disagree", "discuss" and "unrelated") of the news towards a claim. Existing methods tried to tackle the stance detection problem by classification-based algorithms. However, classification-based algorithms make a strong assumption that there is clear distinction between any two stances, which may not be held in the context of stance detection. Accordingly, we frame the detection problem as a ranking problem and propose a rankingbased method to improve detection performance. Compared with the classification-based methods, the ranking-based method compare the true stance and false stances and maximize the difference between them. Experimental results demonstrate the effectiveness of our proposed method.
\end{abstract}

\section{CCS CONCEPTS}

\section{- Computing methodologies $\rightarrow$ Information extraction;}

\section{KEYWORDS}

Fake news; stance detection; learning to rank

\section{ACM Reference Format:}

Qiang Zhang, Emine Yilmaz, and Shangsong Liang. 2018. Ranking-based Method for News Stance Detection. In WWW'18 Companion: The 2018 Web Conference Companion, April 23-27, 2018, Lyon, France, Jennifer B. Sartor, Theo D'Hondt, and Wolfgang De Meuter (Eds.). ACM, New York, NY, USA, 3 pages. https://doi.org/10.1145/3184558.3186919

\section{INTRODUCTION}

Online news on social media is enjoying an increasingly popularity all over the world due to its easy access and wide information dimension. Despite the advantages provided by social media, it also accelerates the spread of fake news or low quality news with intentionally misinformation. Fake news is defined by Facebook as "news articles that purport to be factual, but which contain intentional misstatements of fact with attention to arouse passions, attract viewership, or deceive" [3]. The proliferation of fake news can contribute greatly to people's confusion and distrust. For example, the fake news "Pizzagate" attracted over 1 million tweets by the end of the 2016 United States America presidential election.

This paper is published under the Creative Commons Attribution 4.0 International (CC BY 4.0) license. Authors reserve their rights to disseminate the work on their personal and corporate Web sites with the appropriate attribution.

WWW'18 Companion, April 23-27, 2018, Lyon, France

() 2018 IW3C2 (International World Wide Web Conference Committee), published under Creative Commons CC BY 4.0 License.

ACM ISBN 978-1-4503-5640-4/18/04.

https://doi.org/10.1145/3184558.3186919
Stance detection has been suggested as a crucial first step to detect fake news. ${ }^{1}$ Researchers from both academia and industry initiated the Fake News Challenge (FNC) ${ }^{1}$ and the first stage of FNC (FNC-1) aimed to accelerate the establishment of automatic systems for evaluating the positions that a news source holds about a particular claim. More specifically, given a news headline as a claim and its article body, FNC-1 tried to develop models to estimate the stance of the article body towards its headline. The stance could be one of the labels: "agree", "disagree", "discuss" and "unrelated". All the news with the "agree", "disagree" and "discuss" are assumed as "related". According to the FNC-1, formal definitions of the four stances are as: "Agree" - the body text agrees with the headline; "Disagree" - the body text disagrees with the headline; "Discuss" - the body text discusses the same claim as the headline, but does not take a position; and "Unrelated" - the body text discusses a different claim but not that in the headline.

A GradientBoosting classifier is implemented as the FNC-1 official baseline with a relative score of $75.20 \%$. This classifier makes use of semantic analysis and overlap between headlines and bodies. ${ }^{1}$ As for the FNC-1 submissions, an ensemble of convolutional neural network and gradient-boosted decision trees achieves the highest detection performance ${ }^{2}$. Another ensemble of five multi-layer perceptrons (MLP) achieves a little worse accuracy. These two methods take input of semantic analysis, bag of words and baseline features ${ }^{3}$. Different form the above two methods, the third best team does not try ensemble methods. They use one single MLP with term frequency-inversed document frequency (TF-IDF) as text features [2]. The fourth best team extract both lexical matching and semantic embedding features, and trains another gradient boosting trees ${ }^{4}$. All the aforementioned methods detect stances by classification-based algorithms, the aim of which is to approximate the true probability distribution of the four stances in news articles.

However, classification-based algorithms make a strong assumption that there is clear distinction between any two stances. This assumption may not be hold in the setting of stance detection, as it is challenging to distinguish between any of the two even for humans [1]. Accordingly, we propose a ranking-based method that is more suitable to accomplish the task, as stance should be detected relatively rather than absolutely. We define a ranking loss function in our method to compare the true stance and false stances. Given a news body and its headline, the proposed method applies a multilayer perceptron to produce a value for every stance. Our ranking loss function can maximize the value difference between the true stance and false stances. Experimental results on FNC-1 test dataset validate the effectiveness of our proposed method.

\footnotetext{
${ }^{1}$ http://www.fakenewschallenge.org/.

${ }^{2}$ https://blog.talosintelligence.com/2017/06/talos-fake-news-challenge.html. ${ }^{3}$ https://goo.gl/5qDvPJ.

${ }^{4}$ https://github.com/shangjingbo1226/fnc- 1 .
} 


\section{METHOD}

The proposed model is a single, end-to-end ranking-based algorithm with a multi-layer perceptron. The model input is a pair of headlines and the article bodies. We use stance labels to help compute the value of the objective during the training phase. The multi-layer perceptron with two hidden layers produces a value $u^{+}$for the true stance and three values $u_{1}^{-}, u_{2}^{-}, u_{3}^{-}$for three false stances with the goal to satisfy this constraint: $u^{+} \geq \max \left\{u_{1}^{-}, u_{2}^{-}, u_{3}^{-}\right\}+1$. The objective of the loss function is to maximize the value difference between the true and false stances. During testing, given a news body and its headline the model assigns a particular stance (predicted label) to it, on which the model produces the highest value.

\subsection{Feature Space}

We extract TF-IDF features to represent both headlines and bodies of the news articles: TF-IDF of the headlines, and TF-IDF of the bodies. We also consider cosine similarity between the headline and the body of each article, and those extracted by the official baseline as our features. All features are concatenated to our input vector, denoted as $\mathbf{v}$, that is then fed into the multi-layer perception.

\subsection{Multi-layer Perception}

The multi-layer perception consists of two hidden layers with 100 units and 4 units, respectively. The second hidden layer produces a four-dimensional vector $\mathbf{u}$ with each dimension indicating a value for the corresponding stance respectively,

$$
\mathbf{u}=\mathbf{W}_{2}^{\top} \cdot \operatorname{ReLu}\left(\mathbf{W}_{1}^{\top} \cdot \mathbf{v}+\mathbf{b}_{1}\right)+\mathbf{b}_{2},
$$

where $\mathbf{W}_{1}$ and $\mathbf{W}_{2}$ are the weight matrices, $\mathbf{b}_{1}$ and $\mathbf{b}_{2}$ are the bias vectors in the first and the second hidden layer, respectively; and the rectified linear unit ( $\mathrm{ReLu})$ is applied to the hidden layers as the non-lineaer activation function. Then the ranking loss function can be defined as follows:

$$
L(\mathbf{u})=\max \left(0,1-u^{+}+\max \left(u_{1}^{-}, u_{2}^{-}, u_{3}^{-}\right)\right),
$$

where $u^{+}=u_{i}$ with $i$ being the index of the true stance for the news, and $\left\{u_{1}^{-}, u_{2}^{-}, u_{3}^{-}\right\} \equiv\left\{u_{j}\right\}_{j=1}^{4} /\left\{u_{i}\right\}$. Here $\left\{u_{j}\right\}_{j=1}^{4}$ are the elements in u. The goal of the training is to enlarge the difference between $u^{+}$ and the maximum in $\left\{u_{1}^{-}, u_{2}^{-}, u_{3}^{-}\right\}$for all the news articles.

\subsection{Experimental Setting}

Following the official setting, we split the data set (75,385 news articles) into training, validation and testing subsets. ${ }^{5}$ We apply dropout and include an L2 regularization for the MLP weights in the loss function to mitigate overfitting. We train in mini-batches over the entire training set with the Adadelta optimizer.

The evaluation is based on a weighted, two-layer scoring system. The "related"/"unrelated" classification is given $25 \%$ score weighting in the accuracy evaluation metric because it is easier. The classification between "agree", "disagree" and "discuss" is given $75 \%$ score weighting since it is more difficult and relevant to fake news detection. The scoring system produces a relative score based on the differentially weighted scoring metric.

\footnotetext{
${ }^{5}$ Data set is publicly available from https://github.com/FakeNewsChallenge/fnc- 1 .
}

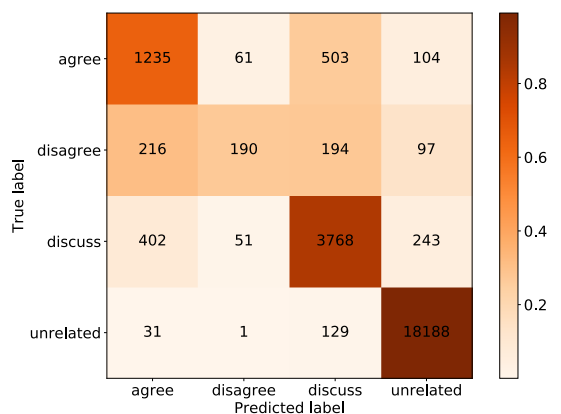

Figure 1: The heat map of detection results.

Table 1: Performance comparison with the state-of-art algo-

\begin{tabular}{|c|c|c|c|c|c|}
\hline \multirow[t]{2}{*}{ Team } & \multicolumn{4}{|c|}{ Accuracy $(\%)$} & \multirow[t]{2}{*}{ Relative Score $(\%)$} \\
\hline & agree & disagree & discuss & unrelated & \\
\hline Official baseline & 9.09 & 1.00 & 79.66 & 97.98 & 75.20 \\
\hline Chips Ahoy! & 55.96 & 0.29 & 70.30 & 98.99 & 80.21 \\
\hline UCLMR & 44.04 & 6.60 & 81.38 & 97.90 & 81.72 \\
\hline Athene & 44.72 & 9.47 & 80.89 & 99.25 & 81.97 \\
\hline Talos & 58.54 & 1.87 & 76.19 & 98.70 & 82.02 \\
\hline This work & 64.90 & 27.26 & 84.41 & 99.12 & 86.66 \\
\hline
\end{tabular}
rithms on the FNC-1 competition dataset.

\section{RESULTS}

As for the FNC-1 official evaluation metric, our method achieves an overall relative score of $86.66 \%$. Fig. 1 demonstrates the performance with a heat map and Table 1 compares our method with the state-ofthe-art. Our method significantly achieves the best performance and improves detection accuracy. When separating "unrelated" pairs of headlines and bodies, we got over $99 \%$ accuracy. Besides, the detection accuracy for "discuss" is improved by $3.72 \%$. Importantly, our method successfully improves the pretty limited accuracy of "agree" and "disagree" by $10.86 \%$ and $187.86 \%$, respectively. This is the most attracting point as "agree" and "disagree" are the most difficult to detect by previous methods and the most relevant to the superordinate goal of automating the stance detection process.

\section{CONCLUSION}

We tackle the news stance detection task using a ranking-based method rather than classification-based algorithms. Given a pair of headlines and article bodies, the ranking-based method can compare and maximize the difference between the true and false stances. Though the architecture is simple, it successfully improves stance detection performance to a large extent.

\section{ACKNOWLEDGMENTS}

This research was supported by the China Scholarship Council (CSC) for award No. 201704910908.

\section{REFERENCES}

[1] William Ferreira and Andreas Vlachos. 2016. Emergent: a novel data-set for stance classification. In Proceedings of the 2016 conference of the North American chapter of the association for computational linguistics: Human language technologies. 11631168.

[2] Benjamin Riedel, Isabelle Augenstein, Georgios P Spithourakis, and Sebastian Riedel. 2017. A simple but tough-to-beat baseline for the Fake News Challenge stance detection task. arXiv preprint arXiv:1707.03264 (2017).

[3] Jen Weedon, William Nuland, and Alex Stamos. 2017. Information operations and Facebook. version 1 (2017), 27. 